\title{
Diseño, construcción y prueba estática experimental de un motor-cohete de combustible solido
} Design, construction and experimental static
testing of a solid rocket motor

\author{
Rafael Robayo-Salazar 1a, Julián Portocarrero-Hermann ${ }^{1 b}$, Juan Meneses-Suta 1c, \\ Jonathan Salgado-Díaz ${ }^{1 d}$, Sergio Martínez-Riaño ${ }^{1 e}$, Fernando Delgado if, \\ Andrea Gómez-Ruge 1g
}

\begin{abstract}
${ }^{1}$ Grupo de Investigación en Estudios Aeroespaciales (GIEA), Programa de Ingeniería Mecánica, Escuela Militar de Aviación (EMAVI) - Fuerza Aérea Colombiana, Colombia. Orcid: ${ }^{a}$ 0000-0003-1687-2885.

Correos electrónicos: ${ }^{\mathrm{a}}$ rafael.robayo@emavi.edu.co, ${ }^{\mathrm{b}}$ julian.portocarrero@emavi.edu.co, c jdmenesess@emavi.edu.co, d laboratorista.pimec@emavi.edu.co, e asistente.pimec@emavi.edu.co,

${ }^{\mathrm{f}}$ fernando.delgado@emavi.edu.co, ${ }^{\mathrm{g}}$ andrea.gomez@emavi.edu.co
\end{abstract}

Recibido: 30 junio, 2020. Aceptado: 21 noviembre, 2020. Versión final: 4 enero, 2021.

\section{Resumen}

Este artículo presenta el diseño, construcción y prueba estática de un prototipo de motor cohete propulsado por combustible sólido tipo Candy. El diseño se basó en la metodología SRM (Solid Rocket Motor Design). El motor cohete incluyó el sistema ignitor, la cámara de combustión, el combustible sólido y la tobera. El diámetro interno de la cámara de combustión y la longitud total del motor cohete fueron de 27,67 mm y $230 \mathrm{~mm}$, respectivamente. La tobera del motor cohete fue de tipo Laval convergente-divergente. El combustible sólido se basó en la mezcla de nitrato de potasio $\left(\mathrm{KNO}_{3}\right)$ (oxidante) y azúcar de alta pureza (o sucrosa: $\mathrm{C}_{12} \mathrm{H}_{22} \mathrm{O}_{11}$ ) (combustible), cuya síntesis se logró a través de un proceso de fusión térmica $\left(150-170^{\circ} \mathrm{C}\right.$ durante $\left.20-25 \mathrm{~min}\right)$. El desempeño del motor cohete fue evaluado mediante una prueba estática de combustión; arrojando como resultados una fuerza de empuje máxima (carga) de 227 $\mathrm{N}$, un impulso total de $98,69 \mathrm{Ns}$ y un impulso especifico de 65,8 s, permitiendo así, su clasificación como un motor cohete clase G. Los resultados obtenidos son considerados de gran importancia en el desarrollo y caracterización de motores cohete de combustible sólido, necesarios para impulsar el campo de la cohetería experimental en Colombia.

Palabras clave: motor cohete; motores de combustión interna; combustible sólido; propelente; prueba estática; combustión; cohetería; cohetes; diseño; industria aeroespacial.

\begin{abstract}
This paper introduces the design, construction, and static testing of a solid rocket motor. The design was based on the solid rocket motor design methodology (SRM). The rocket motor included the igniter system, the combustion chamber, the solid propellant, and the nozzle. The internal diameter of the combustion chamber and the total length of the rocket motor were $27,67 \mathrm{~mm}$ and $230 \mathrm{~mm}$, respectively. The rocket motor nozzle was of the convergent-divergent Laval type. The propellant was based on the mixture of potassium nitrate $\left(\mathrm{KNO}_{3}\right)$ (oxidant) and high purity sugar (or sucrose: $\mathrm{C}_{12} \mathrm{H}_{22} \mathrm{O}_{11}$ ) (fuel), whose synthesis was achieved through a thermal fusion process (150-170 ${ }^{\circ} \mathrm{C}$ for $20-25$ min). The performance of the rocket motor was evaluated using a static combustion test; resulting in a maximum thrust force
\end{abstract}

ISSN impreso: 1657 - 4583. ISSN en línea: 2145 - 8456, CC BY-ND 4.0 @) () 요

Como citar: R. Robayo-Salazar, J. Portocarrero-Hermann, J. Meneses-Suta, J. Salgado-Díaz, S. Martínez-Riaño, F. Delgado, A. Gómez-Ruge, "Diseño, construcción y prueba estática experimental de un motor-cohete de combustible solido," Rev. UIS Ing., vol. 20, no. 2, pp. 97-108, 2021, doi: 10.18273/revuin.v20n2-2021009 
(load) of $227 \mathrm{~N}$, a total impulse of 98,69 Ns and a specific impulse of 65,8 s, thus allowing its classification as a class $\mathrm{G}$ rocket motor. The results obtained are considered of great importance in the development and characterization of solid rocket motors, necessary to promote the experimental rocketry in Colombia.

Keywords: rocket motor; internal combustion engines; solid propellant; static test; combustion; rocketry; rockets; rocket thrust; design; aerospace industry.

\section{Introducción}

Los cohetes experimentales son considerados por la NASA como una herramienta única para llevar a cabo investigaciones científicas en regiones importantes del espacio que son demasiado bajas para ser muestreadas por satélites [1]. En este sentido, los cohetes experimentales son empleados para el transporte, a bajo costo, de instrumentos científicos de medición y observación (cargas útiles), los cuales incluyen un sistema de recuperación. Entre los campos de aplicación se han reportado la bio-meteorología, fotobiología atmosférica preventiva, monitoreo de contaminación, telecomunicaciones, observación de territorios y toma de imágenes aéreas, entre otras [2]. Además del aporte a la ciencia y la tecnología, las misiones de cohetería experimental también proporcionan herramientas invaluables para la formación académica y científica [3], promoviendo, a través del desarrollo de proyectos de investigación, el surgimiento de escuelas de conocimiento necesarias para avanzar hacia misiones más costosas y de mayor duración [4], como el lanzamiento de cohetes tipo sonda y la colocación en posiciones suborbitales y orbitales de nano y picosatélites al servicio del país [5].

El alcance o apogeo de un cohete experimental depende principalmente del desempeño y capacidad de empuje y/o propulsión del motor cohete. El motor cohete es un motor de combustión interna que no toma del exterior los gases que debe acelerar para generar el empuje, sino que tiene todo lo necesario en su interior, es decir, se trata de sistemas autónomos, que no dependen del medio que los rodea [6].

Dentro de los tipos de motor cohete comúnmente utilizados se destacan los químicos, los cuales aprovechan la reacción química de distintos compuestos en una cámara de combustión, de forma que se generan gases de alta temperatura y presiones muy altas [7].

Estos gases son entonces acelerados por una tobera de tipo convergente-divergente y son expulsados a gran velocidad para producir el cambio de momentum que impulsa al cohete. Adicional al empuje por momentum, se suma el empuje por presión, que resulta de la diferencia de presiones existentes entre los gases de escape y la atmosfera, en la salida de la tobera. A la mezcla de los compuestos químicos se le llama propelente (combustible) y a este tipo de motores se les clasifica según el estado físico del mismo en; propelente líquido, propelente sólido, propelente gaseoso y propelente hibrido [8], [9].

En el caso particular de los propelentes sólidos, los combustibles tipo Candy han sido utilizados con éxito en el campo de la cohetería experimental [10], [11]. Estos combustibles se basan en la combinación de nitrato de potasio $\left(\mathrm{KNO}_{3}\right)$ (oxidante) y azúcares (combustible). Los tipos de propelentes Candy más comunes son los derivados de las mezclas: nitrato de potasio/sucrosa (KNSU), nitrato de potasio/sorbitol (KNSO) y nitrato de potasio/dextrosa (KNDX).

La composición ideal propuesta por Richard Nakka para este tipo de propelentes es de $65 \%$ oxidante y $35 \%$ combustible (en masa) [12]. Independientemente del tipo de combustible utilizado, los motores cohete son clasificados de acuerdo con su capacidad de propulsión (impulso especifico e impulso total). La Tabla 1 relaciona el impulso total $\left(\mathrm{I}_{\mathrm{T}}\right)$ para las diferentes clases de motores cohete.

En Colombia, los desarrollos en el campo de la cohetería experimental se han concentrado en la investigación de motores cohetes propulsados por combustibles sólidos [13]. Al respecto, investigadores de la Universidad San Buenaventura reportaron el diseño de un motor cohete clase G y otro de $800 \mathrm{~N}$ de fuerza de empuje (teórica), ambos basados en un combustible tipo KNSB [14], [15]. Por su parte, la Universidad Militar Nueva Granada llevo a cabo el diseño conceptual de un motor cohete clase I [16], [17]. Investigadores de la Universidad de los Andes reportaron el diseño de un motor cohete tipo KNSB de $210 \mathrm{~N}$ de fuerza de empuje (teórica) [18]. Así mismo, la Fundación Universitaria los Libertadores definió el diseño conceptual de un motor cohete de $2,7 \mathrm{KN}$ de fuerza de empuje [19], [20]. Trabajos complementarios también han sido reportados por la Universidad Nacional de Colombia [21], Universidad Libre [22], Universidad Industrial de Santander [23]; a los que se ha sumado, en algunos casos, la participación de la Industria Militar Colombiana (INDUMIL) y de la Fuerza Aérea Colombiana (FAC). Cabe destacar que la mayoría de 
estos reportes corresponden a diseños de tipo conceptual (teóricos), y la validación de sus desempeños mediante pruebas reales (estáticas) de combustión, o lanzamientos del cohete, no ha sido reportada, lo cual impide su transferencia tecnológica.

Tabla 1. Clasificación de motores cohete según su capacidad de propulsión

\begin{tabular}{|c|c|c|c|}
\hline \multirow{2}{*}{ Clase } & \multicolumn{3}{|c|}{ Impulso total $\left(\mathbf{I}_{\mathbf{T}}\right)$} \\
\hline & \multicolumn{3}{|c|}{$(\mathbf{N s})$} \\
\hline A & 1,26 & - & 2,5 \\
\hline $\mathrm{B}$ & 2,5 & - & 5 \\
\hline $\mathrm{C}$ & 5 & - & 10 \\
\hline $\mathrm{D}$ & 10 & - & 20 \\
\hline$E$ & 20 & - & 40 \\
\hline $\mathrm{F}$ & 40 & - & 80 \\
\hline $\mathrm{G}$ & 80 & - & 160 \\
\hline $\mathrm{H}$ & 160 & - & 320 \\
\hline $\mathrm{I}$ & 320 & - & 640 \\
\hline $\mathrm{J}$ & 640 & - & 1.280 \\
\hline $\mathrm{K}$ & 1.280 & - & 2.560 \\
\hline $\mathrm{L}$ & 2.560 & - & 5.120 \\
\hline $\mathrm{M}$ & 5.120 & - & 10.240 \\
\hline $\mathrm{N}$ & 10.240 & - & 20.480 \\
\hline $\mathrm{O}$ & 20.480 & - & 40.960 \\
\hline $\mathrm{P}$ & 40.960 & - & 81.920 \\
\hline $\mathrm{Q}$ & 81.920 & - & 163.840 \\
\hline $\mathrm{R}$ & 163.840 & - & 327.680 \\
\hline $\mathrm{S}$ & 327.680 & - & 655.360 \\
\hline $\mathrm{T}$ & 655.360 & - & $1 ’ 310.720$ \\
\hline $\mathrm{U}$ & 1'310.720 & - & $2^{\prime} 621.440$ \\
\hline $\mathrm{V}$ & 2'621.440 & - & 5'242.880 \\
\hline
\end{tabular}

Fuente: Adaptada de [12].

Recientemente, Colombia planteó su política de desarrollo espacial a través del CONPES 3983 del 13 de enero del 2020; por medio del cual se establecen las condiciones habilitantes para que las tecnologías aeroespaciales sean un impulsor de la productividad, la diversificación y sofisticación de todo el sector productivo colombiano [24]. En este propósito, el desarrollo de proyectos en el área de cohetería es identificado como una línea estratégica para finalmente lograr el posicionamiento de nanosatélites al servicio de Colombia. Esta investigación se desarrolló en el marco del proyecto "FACSON-1" del Programa de Ingeniería Mecánica (PIMEC) de la Escuela Militar de Aviación "Marco Fidel Suarez" (EMAVI) - Fuerza Aérea Colombiana (FAC), el cual tiene como propósito el lanzamiento de un prototipo de cohete experimental basado en tecnología colombiana. Con relación a lo anterior, el objetivo de este artículo es presentar el diseño, construcción y prueba estática de combustión de un prototipo a escala del motor cohete.

\section{Materiales y metodología}

La metodología desarrollada en esta investigación se resume en tres fases (Figura 1): la primera, relacionada con el diseño y construcción del motor cohete; la segunda, con la síntesis y moldeo del propelente sólido; y finalmente, la tercera, asociada con la prueba estática del motor cohete. El diseño conceptual del motor cohete se realizó con base en la metodología SRM (Solid Rocket Motor Design) propuesta por Richard Nakka [12]. La clase del motor seleccionada para fines de diseño se estableció con base en el logro de un impulso total de 80160 Ns (motor clase G).

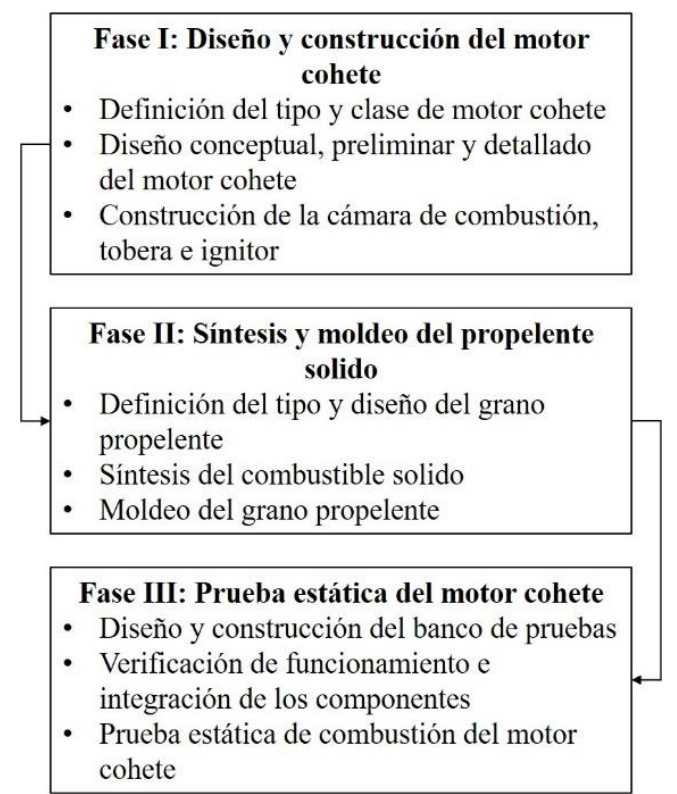

Figura 1. Metodología experimental desarrollada en la investigación. Fuente: elaboración propia.

El diseño preliminar y detallado del motor cohete se realizó mediante el uso del software CAD (diseño asistido por computadora) SolidWorks, obteniendo un modelo 3D para cada uno de los componentes y un plano para su posterior fabricación. Para el mecanizado de la cámara de combustión y la tobera del motor cohete se utilizó acero galvanizado y acero de bajo contenido de carbono AISI 1020, respectivamente. La síntesis del combustible sólido tipo Candy se basó en la combinación de nitrato de potasio $\left(\mathrm{KNO}_{3}\right)$ (oxidante) y azúcar de alta pureza (o sucrosa: $\mathrm{C}_{12} \mathrm{H}_{22} \mathrm{O}_{11}$ ) (combustible); componentes que inicialmente fueron sometidos a un proceso de molienda fina en un molino de bolas y posteriormente fueron mezclados y homogenizados en seco. 
El tamaño de partícula promedio obtenido es inferior a la malla \#200 $(75 \mu \mathrm{m})$. La obtención del grano propelente se realizó mediante fusión térmica de la mezcla a una temperatura controlada de entre $150-170^{\circ} \mathrm{C}$ durante 20 $25 \mathrm{~min}$.

El desempeño del motor cohete fue evaluado mediante una prueba estática de combustión en un banco de pruebas que acoplaba una celda de carga de punto único de $70 \mathrm{~kg}$ de capacidad, un sistema de encendido programado y un sistema electrónico de control y adquisición de datos (carga vs. tiempo). Durante la prueba de combustión estática fue posible obtener la fuerza de empuje generada por el motor-cohete, permitiendo su caracterización y posterior clasificación. El sistema electrónico de control y adquisición de datos se integró a partir de una tarjeta de desarrollo electrónica y programable Arduino I que incluía: un microcontrolador, un módulo micro-SD para el almacenamiento de datos, un módulo HX711 como interfaz para la adquisición de los datos de la celda de carga, cuatro diodos LED indicadores de estado, un pulsador para inicio de la prueba y un relé $5 \mathrm{~V} \mathrm{DC}$. El sistema electrónico fue programado en la plataforma de Arduino en un lenguaje de programación de alto nivel (Processing).

La metodología SRM (Solid Rocket Motor Design) propuesta por Richard Nakka [12], seguida para el diseño conceptual del motor cohete de combustible sólido, consiste en un procedimiento secuencial que permite la selección y cálculo de las variables teóricas involucradas en el diseño de la cámara de combustión, grano propelente y tobera, permitiendo finalmente su dimensionamiento para un desempeño deseado (Figura 2 ), que en este caso corresponde a un motor cohete clase G.

Donde,

$\mathrm{D}_{\mathrm{c}}$ : diámetro de la cámara

$\mathrm{L}_{\mathrm{c}}$ : longitud de la cámara

$\mathrm{V}_{\mathrm{c}}$ : volumen de la cámara

$\mathrm{D}_{\mathrm{o}}$ : diámetro externo del grano propelente

do: diámetro del núcleo del grano

Lo: longitud del grano propelente

$\mathrm{A}_{\mathrm{e}}$ : área de salida de gases

$\mathrm{A}_{\mathrm{t}}$ : área de la garganta de la tobera

Lc: longitud del área convergente de la tobera

Ld: longitud área divergente de la tobera

Lo: longitud de la tobera

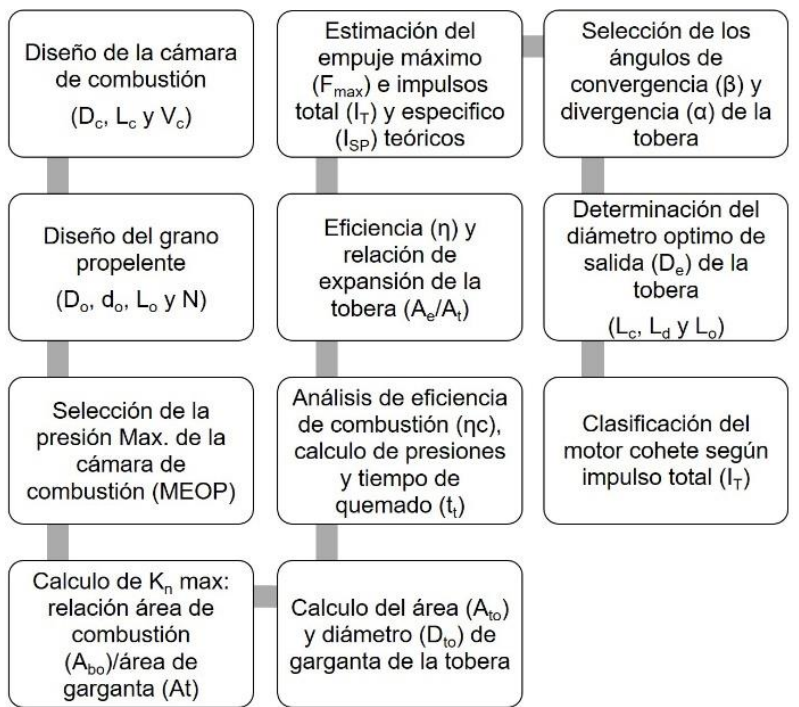

Figura 2. Metodología SRM (Solid Rocket Motor Design). Fuente: elaboración propia.

\section{Resultados y análisis}

\subsection{Diseño y construcción del motor cohete}

El diseño y construcción del motor cohete incluyo; la cámara de combustión, el sistema ignitor y la tobera o boquilla de escape de los gases. Para el diseño preliminar y detallado de los componentes se utilizó el software CAD SolidWorks, obteniendo un modelo 3D y un plano de cada uno de los componentes para su posterior fabricación. Estos diseños fueron posibles tras la aplicación de la metodología SRM (Solid Rocket Motor Design) propuesta por Richard Nakka [12]. Inicialmente, se diseñó la cámara de combustión, cuyas dimensiones están directamente relacionadas al diseño conceptual del grano propelente. Con relación a lo anterior, el grano propelente fue diseñado de forma cilíndrica para encajar perfectamente dentro de la cámara de combustión del motor cohete, buscando la mayor eficiencia volumétrica.

En este sentido, la cámara de combustión corresponde a un tubo galvanizado y roscado en los extremos de 27,7 $\mathrm{mm}$ de diámetro interno $\left(\mathrm{D}_{\mathrm{c}}\right), 5,5 \mathrm{~mm}$ de espesor de pared y $152,4 \mathrm{~mm}$ de longitud $\left(\mathrm{L}_{\mathrm{c}}\right)$, tal y como se puede observar en la Figura 3. De acuerdo con estas dimensiones, la cámara de combustión presento un volumen $\left(\mathrm{V}_{\mathrm{c}}\right)$ de $91.642 \mathrm{~mm}^{3}$.

El ignitor se acoplo a la cámara de combustión en el extremo opuesto a la tobera por medio de un tapón roscado (Figura 3). El ignitor consistió en un cartucho de Candy KNSU en contacto con un filamento de acero como resistencia eléctrica. 
Mediante el sistema electrónico de control se programó el encendido del ignitor aplicando $12 \mathrm{~V}$ y $100-200 \mathrm{~mA}$ de corriente continua, logrando que el filamento de acero (resistencia eléctrica) alcanzara la temperatura umbral $\left(\geq 300^{\circ} \mathrm{C}\right)$. En el centro del tapón ignitor se dispone un orificio de $1 \mathrm{~mm}$ a través del cual pasan los cables de alimentación que están conectados al alambre resistivo encapsulado en el cartucho de Candy KNSU.

Luego de fijar el circuito, el tapón fue sellado internamente, con un compuesto de tipo ablativo basado en la mezcla de resina poliéster, partículas refractarias y fibra de vidrio, para evitar fugas y pérdida de presión durante la combustión.

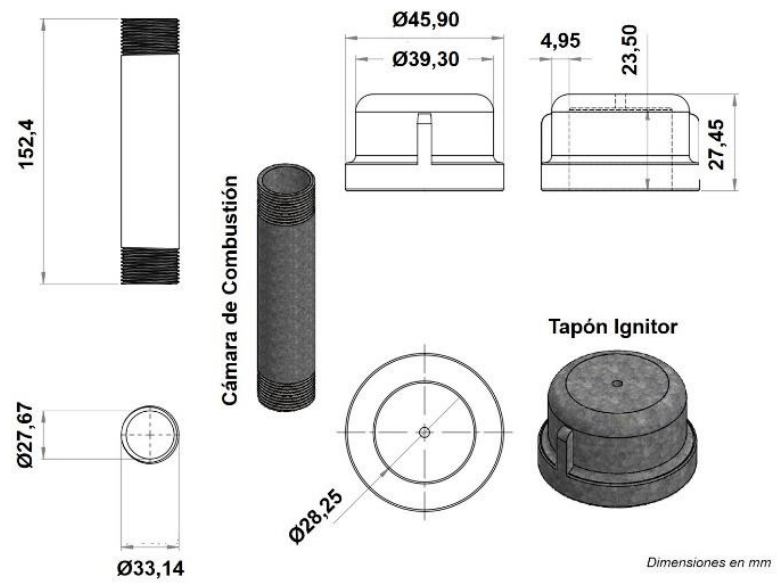

Figura 3. Diseño de la cámara de combustión del motor cohete: planos de fabricación. Fuente: elaboración propia.

La tobera diseñada para el motor cohete corresponde a una de tipo convergente-divergente o tobera de Laval, cuyo perfil geométrico varía el área seccionada transversalmente (o diámetro). La condición de diseño de la tobera se realizó con un área de entrada $\left(A_{e}\right)$ (convergente), área de garganta $\left(\mathrm{A}_{\mathrm{t}}\right)$ y área de salida $\left(\mathrm{A}_{\mathrm{e}}\right)$ (divergente) de 601,3, 33,9 y $339,0 \quad \mathrm{~mm}^{2}$, respectivamente. Para este diseño, el diámetro de la garganta de la tobera fue de $6,57 \mathrm{~mm}$, con una relación de expansión optima $A_{e} / A_{t}$ de 10 y ángulos convergentedivergente de $60-24^{\circ}$. La relación entre el área de quemado y el área de la garganta de la tobera $\left(\mathrm{K}_{\mathrm{n}}(\max )\right)$ fue de 254 . La longitud de la tobera $\left(\mathrm{L}_{\mathrm{o}}\right)$, representada como la suma de las longitudes convergente $\left(\mathrm{L}_{\mathrm{c}}\right)$ y divergente $\left(\mathrm{L}_{\mathrm{d}}\right)$, fue de 51,68 $\mathrm{mm}$ con un espesor de pared constante en todas las zonas de 2,36 $\mathrm{mm}$. La geometría presentada en la Figura 4 fue obtenida por mecanizado de un bloque macizo de acero de bajo contenido de carbono AISI 1020 en un torno CNC de 4 ejes.

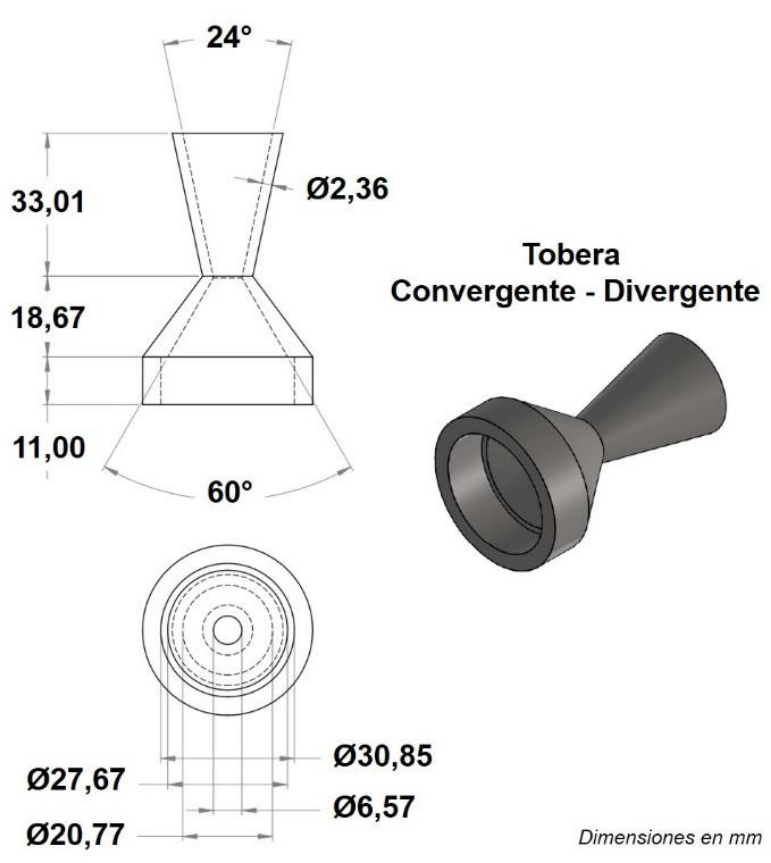

Figura 4. Diseño de la tobera del motor cohete: planos de fabricación. Fuente: elaboración propia.

\subsection{Síntesis y moldeo del combustible sólido KNSU (grano propelente)}

El propelente solido se basó en la mezcla de dos constituyentes principales: un oxidante y un combustible. En el caso de los combustibles sólidos tipo Candy, el oxidante comúnmente utilizado para su síntesis es el nitrato de potasio $\left(\mathrm{KNO}_{3}(\mathrm{KN})\right)$. Entre las azúcares (combustible) se destacan: la sucrosa (SU), el sorbitol (SO) y la dextrosa (DX). La composición ideal propuesta por Richard Nakka para este tipo de propelentes es de $65 \%$ oxidante y $35 \%$ combustible (en masa) [12]. En este sentido, la mezcla estándar seleccionada para la síntesis y moldeo del grano propelente fue $65 \% \mathrm{KNO}_{3}+35 \% \mathrm{SU}$ (Candy tipo KNSU).

Respecto al diseño del grano propelente cabe anotar que, el motor cohete opera bajo el principio de convertir energía calórica, de las reacciones químicas, en energía cinética. Es decir, el calor liberado por la combustión del propelente provee la energía calórica; la alta velocidad de los productos de escape saliendo del motor a través de la tobera otorgan la energía cinética. Por lo tanto, la combustión del propelente KNSU es una reacción química exotérmica $\left(\approx 1.450^{\circ} \mathrm{C}\right)$. Para iniciar la combustión se necesitó de una fuente de calor externa (sistema ignitor) que suministrara la energía necesaria a un nivel de umbral (temperatura $\geq 300^{\circ} \mathrm{C}$ ). 
En efecto, la combustión del propelente tipo KNSU está dada por la ecuación 1 (reactivos $\rightarrow$ productos) $(1 \mathrm{~mol} \mathrm{de}$ sucrosa y 6,29 moles de $\mathrm{KNO}_{3}$ ) [25].

$$
\begin{aligned}
\mathrm{C}_{12} \mathrm{H}_{22} \mathrm{O}_{11}+6,29 & \mathrm{KNO}_{3} \\
& \rightarrow 3,80 \mathrm{CO}_{2}+5,21 \mathrm{CO} \\
& +7,79 \mathrm{H}_{2} \mathrm{O}+3,07 \mathrm{H}_{2} \\
& +3,14 \mathrm{~N}_{2}+3 \mathrm{~K}_{2} \mathrm{CO}_{3} \\
& +0,27 \mathrm{KOH}
\end{aligned}
$$

Donde los compuestos se simbolizan de la siguiente manera: sucrosa (solido) $\left(\mathrm{C}_{12} \mathrm{H}_{22} \mathrm{O}_{11}\right)$, nitrato de potasio (solido) $\left(\mathrm{KNO}_{3}\right)$, dióxido de carbono (gas) $\left(\mathrm{CO}_{2}\right)$, monóxido de carbono (gas) (CO), vapor (gas) $\left(\mathrm{H}_{2} \mathrm{O}\right)$, hidrogeno (gas) $\left(\mathrm{H}_{2}\right)$, nitrógeno (gas) $\left(\mathrm{N}_{2}\right)$, carbonato de potasio (liquido) $\left(\mathrm{K}_{2} \mathrm{CO}_{3}\right)$ e hidróxido de potasio (liquido) (KOH).

El grano propelente consistió en un solo segmento cilíndrico de $150 \mathrm{~mm}$ de longitud $\left(\mathrm{L}_{\mathrm{o}}\right)$ y $27,67 \mathrm{~mm}$ de diámetro $\left(D_{0}\right)$ (Figura 5), con un núcleo hueco central que se extiende a través de todo el grano para incrementar el área de la superficie del propelente expuesta a la combustión inicial. La forma del núcleo hueco fue de tipo tubular o circular con un diámetro $\left(\mathrm{d}_{0}\right)$ de $6,35 \mathrm{~mm}$. Cabe resaltar que el empuje que el motor genera es proporcional al área en combustión en cualquier instante particular del tiempo, que depende casi exclusivamente de la forma inicial del grano [25]. Este grano cilíndrico hueco y el sistema ignitor del motor cohete fueron diseñados para promover el quemado radial y un perfil de empuje-tiempo de tipo progresivo. Este quemado empieza a lo largo de la longitud del núcleo central, con el avance progresivo de la superficie de la quema en retroceso y radialmente hacia el exterior. La relación longitud/diámetro (L/D) del grano propelente fue de 5,42 .

Para el diseño del motor cohete, siguiendo la metodología SRM [12], se tuvo en cuenta una fracción de carga volumétrica del motor cohete de 0,932 , definida como la relación entre el volumen del grano $\left(\mathrm{V}_{\mathrm{g}}\right)$ y el volumen de la cámara $\left(\mathrm{V}_{\mathrm{c}}\right)$. Así entonces, el volumen del grano propelente fue de $85.448 \mathrm{~mm}^{3}$, con una masa de $0,153 \mathrm{~kg}$ calculada para una densidad real de $1,79 \mathrm{~g} / \mathrm{cm}^{3}$. El área total de quemado $\left(\mathrm{A}_{\mathrm{bo}}\right)$ del combustible sólido fue de $6.410 \mathrm{~mm}^{2}$, con una generación de presión teórica (MEOP) en la cámara de combustión de 850 psi. Al respecto, la cámara de combustión de acero galvanizado diseñada tiene la capacidad de soportar este nivel de presión.

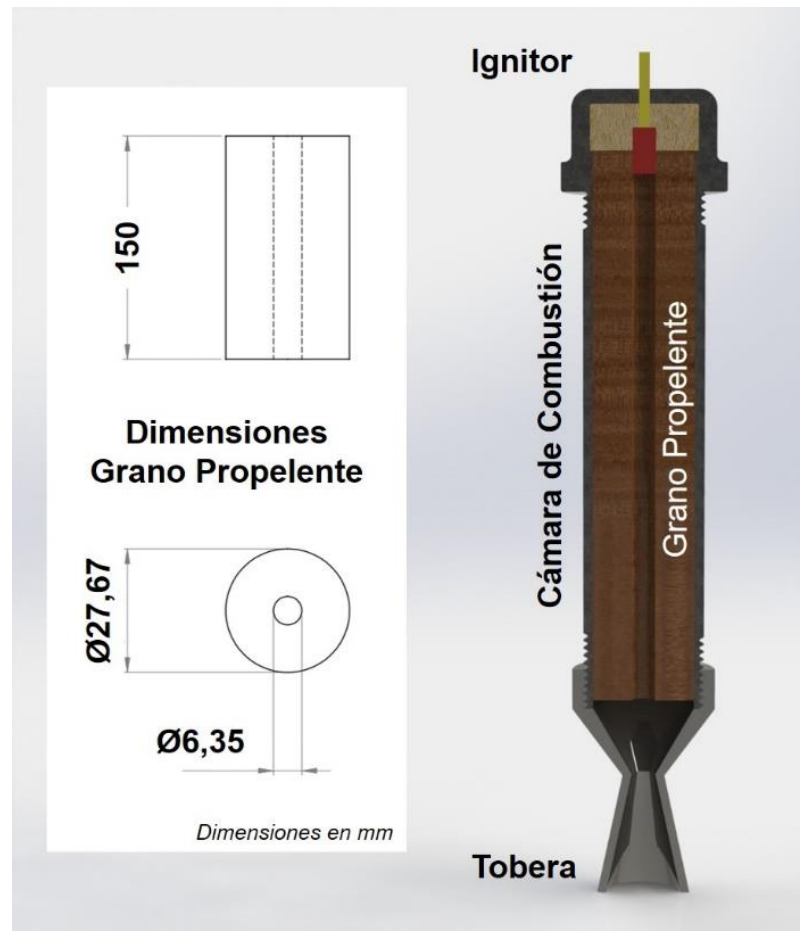

Figura 5. Diseño del motor cohete y dimensiones del grano propelente. Fuente: elaboración propia.

Cabe destacar que el proceso de moldeo del grano propelente se llevó a cabo mediante la fusión térmica de la mezcla $65 \% \mathrm{KNO}_{3}-35 \% \mathrm{SU}$ (KNSU) y su posterior vaciado dentro de la cámara de combustión. Inicialmente, como se puede observar en la Figura 6, la mezcla KNSU fue calentada progresivamente hasta alcanzar su estado fundido a una temperatura controlada de $150-170^{\circ} \mathrm{C}$. El control de la temperatura se llevó a cabo utilizando una cámara termográfica FLIR E40. La fusión térmica de la mezcla KNSU se logró en un tiempo de entre 20-25 min; tiempo durante el cual la mezcla se mantuvo en constante agitación manual.

Cabe destacar que la temperatura alcanzada en este proceso está por encima del punto de fusión de la sucrosa y esta tiende a "caramelizarse", mientras que el $\mathrm{KNO}_{3}$ permanece como partículas sólidas parcialmente disueltas y suspendidas en la matriz de sucrosa. La "caramelización" de la mezcla de Candy se caracteriza por un oscurecimiento de la masa fundida, tal y como se puede observar en la Figura 6. Al respecto, como resultado de varios ensayos preliminares se estableció que un color oscuro implica que la mezcla se ha calentado demasiado tiempo a una temperatura demasiado alta. 


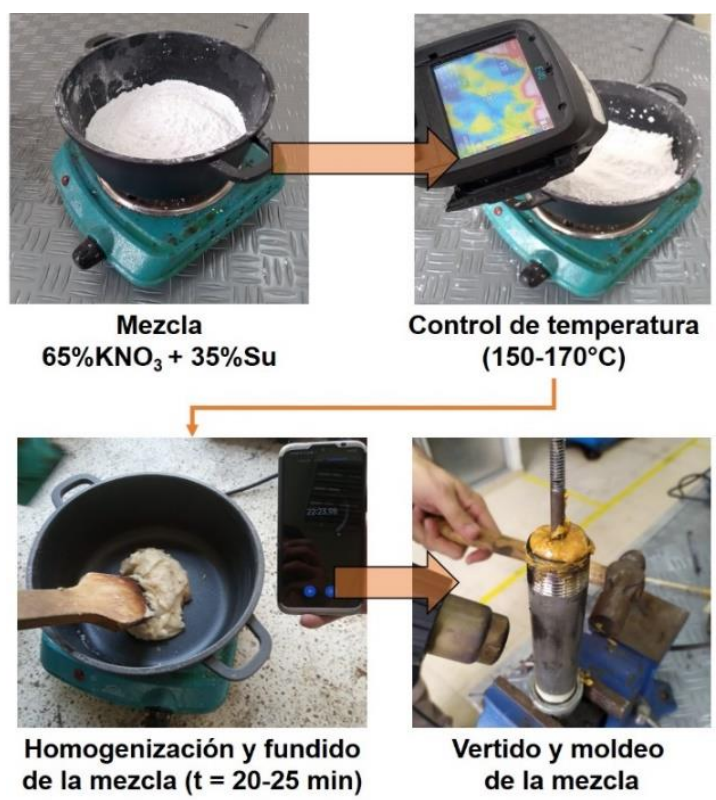

Figura 6. Proceso de síntesis y moldeo del grano propelente (combustible sólido tipo Candy). Fuente: elaboración propia.

Una vez la mezcla se encontraba en estado fundido, fue homogenizada y vertida dentro de la cámara de combustión (molde). La aplicación de vibrado mecánico favoreció la extracción del aire atrapado durante el vaciado de la mezcla de Candy. De manera previa y durante el proceso de moldeo del grano propelente, la cámara de combustión fue calentada $\left(150{ }^{\circ} \mathrm{C}\right)$ externamente utilizando una pistola de calor (Figura 6); esto favoreció el vertido y la compactación de la mezcla dentro del molde (cámara de combustión), impidiendo su rápida solidificación. Cabe anotar que, el núcleo hueco tubular (o circular) del grano propelente se obtuvo fijando una varilla de acero de $6,35 \mathrm{~mm}$ de diámetro de forma concéntrica al interior de la cámara de combustión. Una vez el grano propelente solidifico $(\approx 60 \mathrm{~min})$, la varilla central fue retirada, logrando la geometría de diseño requerida. Luego de desmoldar el núcleo, la cámara de combustión con el grano propelente en su interior se conservó sellado con una película plástica, con la finalidad de preservarlos de la humedad hasta el momento de realizar la prueba estática de combustión.

\subsection{Prueba estática del motor cohete}

La prueba estática de combustión permitió la validación del diseño y la clasificación del motor cohete en función de su desempeño. Inicialmente, se realizó el ensamble y verificación de todos los componentes que integran el motor cohete (Figura 7).
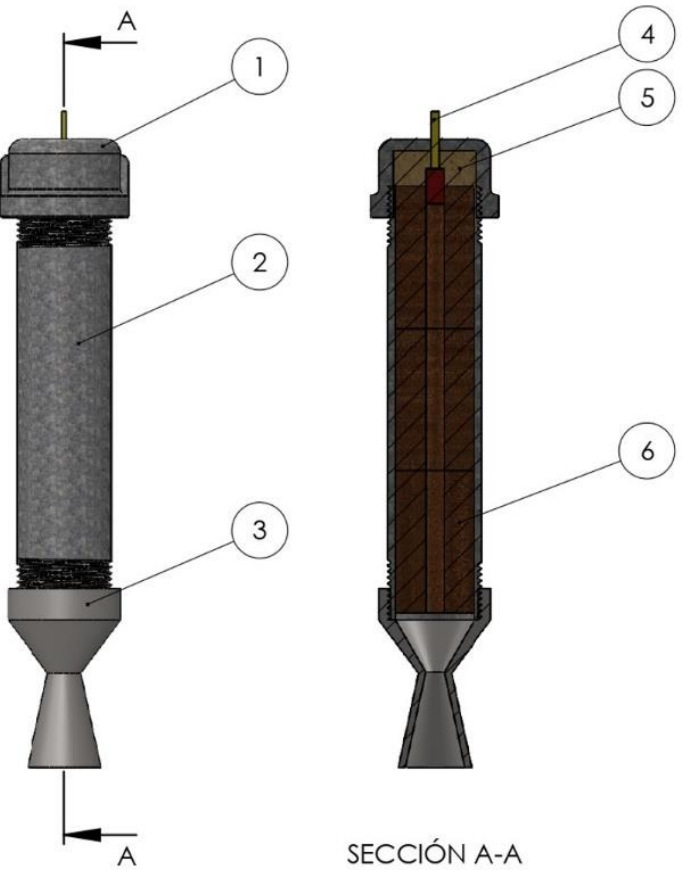

Figura 7. Descripción motor cohete: 1) Tapón ignitor de acero galvanizado de 1", 2) Tubo acero galvanizado

(cámara de combustión) de 1"x 6", 3) Tobera convergente-divergente, 4) Ignitor electrónico, 5) Sellante material compuesto y 6) Grano propelente.

Fuente: elaboración propia.

La Figura 8 presenta el montaje experimental de la prueba estática de combustión del motor cohete, el cual fue colocado de forma horizontal apoyando un tornillo que fue fijado al tapón ignitor, sobre la celda de carga de punto único de $70 \mathrm{~kg}$ de capacidad. El banco de pruebas cuenta con un sistema guía que permite el libre deslizamiento horizontal del motor cohete. La fuerza de empuje (carga) y el tiempo de propulsión fueron los datos primarios recolectados durante la prueba. La adquisición de estos datos fue posible mediante la adaptación de un sistema electrónico (interfaz) que controlaba: 1) la ignición programada del motor cohete y 2) la captación y almacenamiento simultaneo de los datos (carga vs. tiempo) durante la prueba. Previo a la ignición del motor cohete, se llevaron a cabo pruebas de verificación de los sistemas de encendido y adquisición de datos, para confirmar el correcto funcionamiento de todos los subsistemas.

Los resultados obtenidos mediante la prueba estática de combustión se presentan en la Tabla 2 y Figura 9. Es posible identificar que el perfil empuje-tiempo obtenido fue de tipo progresivo, característico de un grano propelente con núcleo hueco tubular (o circular), con un 
valor de empuje máximo $\left(\mathrm{F}_{\max }\right)$ registrado de $227 \mathrm{~N}$. Aunque este valor de empuje es un criterio importante para caracterizar la capacidad de elevación de un motor cohete, no proporciona ninguna indicación de que tan alto será propulsado el cohete. Para conocer esto, se necesita una medida de la producción total en términos de capacidad de propulsión. El criterio esencial para esto es el impulso total $\left(\mathrm{I}_{\mathrm{T}}\right)$ del motor cohete, que relaciona el empuje con el tiempo o duración de la propulsión. Cabe destacar que el apogeo de un cohete también depende de la relación empuje/masa del cohete.

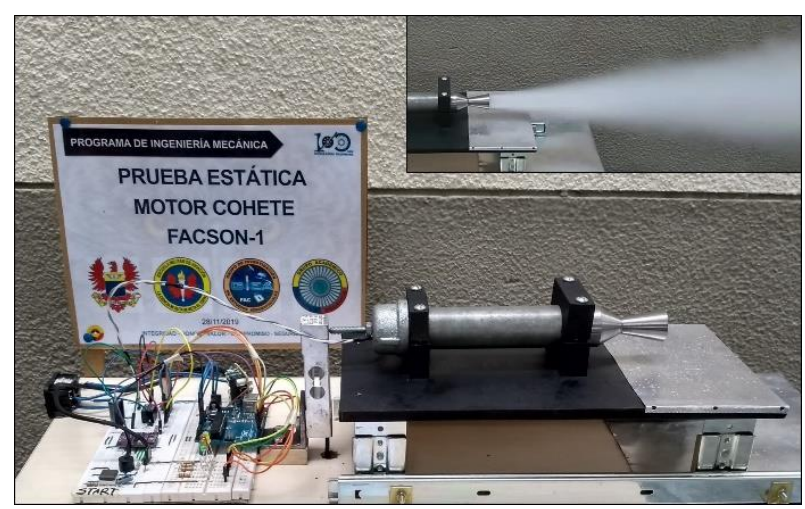

Figura 8. Montaje y prueba estática de combustión del motor cohete. Fuente: elaboración propia.

Los resultados obtenidos mediante la prueba estática de combustión se presentan en la Tabla 2 y Figura 9. Es posible identificar que el perfil empuje-tiempo obtenido fue de tipo progresivo, característico de un grano propelente con núcleo hueco tubular (o circular), con un valor de empuje máximo $\left(\mathrm{F}_{\max }\right)$ registrado de $227 \mathrm{~N}$. Aunque este valor de empuje es un criterio importante para caracterizar la capacidad de elevación de un motor cohete, no proporciona ninguna indicación de que tan alto será propulsado el cohete. Para conocer esto, se necesita una medida de la producción total en términos de capacidad de propulsión. El criterio esencial para esto es el impulso total $\left(\mathrm{I}_{\mathrm{T}}\right)$ del motor cohete, que relaciona el empuje con el tiempo o duración de la propulsión. Cabe destacar que el apogeo de un cohete también depende de la relación empuje/masa del cohete.

A partir de la curva carga (N) vs. tiempo (s) obtenida por medio de la prueba estática fue posible determinar el impulso total $\left(\mathrm{I}_{\mathrm{T}}\right)$ del motor cohete. El impulso total $\left(\mathrm{I}_{\mathrm{T}}\right)$ se define como la integral del empuje (F) durante el tiempo de propulsión (t) del motor (ecuación 2):

$$
I_{T}=\int_{0}^{t} F d t
$$

Tabla 2. Datos de carga $(\mathrm{N})$ registrados por medio de la prueba estática de combustión

\begin{tabular}{|c|c|}
\hline Tiempo (s) & Carga (N) \\
\hline 0,0 & 0,00 \\
\hline 0,1 & 0,00 \\
\hline 0,2 & 0,00 \\
\hline 0,3 & 0,00 \\
\hline 0,4 & 0,49 \\
\hline 0,5 & 23,05 \\
\hline 0,6 & 34,42 \\
\hline 0,7 & 109,15 \\
\hline 0,8 & 156,91 \\
\hline 0,9 & 227,22 \\
\hline 1,0 & 218,59 \\
\hline 1,1 & 85,71 \\
\hline 1,2 & 53,64 \\
\hline 1,3 & 24,12 \\
\hline 1,4 & 17,65 \\
\hline 1,5 & 13,53 \\
\hline 1,6 & 4,71 \\
\hline 1,7 & 5,10 \\
\hline 1,8 & 5,59 \\
\hline 1,9 & 4,51 \\
\hline 2,0 & 4,32 \\
\hline
\end{tabular}

Fuente: elaboración propia.

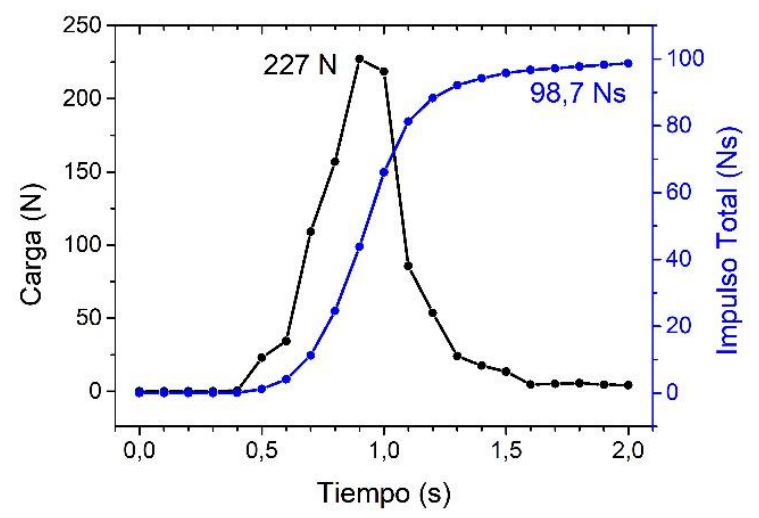

Figura 9. Gráfico Carga (N) vs. Tiempo (s) e Impulso Total (Ns) obtenido a partir de la prueba estática de combustión del motor cohete. Fuente: elaboración propia.

De acuerdo con los datos reportados en la Figura 9, el impulso total $\left(\mathrm{I}_{\mathrm{T}}\right)$ del motor cohete fue de 98,7 Ns. A partir de este resultado y la clasificación de los motores cohetes presentada en la Tabla 1, este motor cohete puede ser catalogado como un motor clase $\mathrm{G}$ con un impulso total $\left(\mathrm{I}_{\mathrm{T}}\right)$ entre 80-160 Ns. Con base en el impulso total $\left(\mathrm{I}_{\mathrm{T}}\right)$ obtenido, y teniendo en cuenta que el tiempo de 
propulsión $(t)$ fue de 1,3 s, fue determinado el empuje promedio $\left(\mathrm{F}_{\mathrm{avg}}\right)$ del motor cohete utilizando la ecuación 3. Este calculo arrojo como resultado un valor de $\mathrm{F}_{\text {avg }}$ de $75,9 \mathrm{~N}$

$$
F_{\text {avg }}=\frac{I_{T}}{t}=\frac{98,7 \mathrm{Ns}}{1,3 \mathrm{~s}}=75,9 \mathrm{~N}
$$

Por su parte, el impulso especifico ( $\left.\mathrm{I}_{\mathrm{SP}}\right)$ es una medida del rendimiento (potencial) de los combustibles sólidos y está definido como el impulso total impartido por unidad de gasto (peso o volumétrico) de propelente utilizado. En este sentido, un mayor valor de impulso especifico ( $\mathrm{I}_{\mathrm{SP}}$ ) indica un propelente eficiente y de alto poder. El cálculo del impulso especifico $\left(\mathrm{I}_{\mathrm{SP}}\right)$ está representado con la ecuación 4 , siendo $m$ la masa del grano propelente y $g$ la constante gravitacional. Con base en lo anteriormente descrito, el impulso especifico $\left(\mathrm{I}_{\mathrm{SP}}\right)$ del motor cohete fue de $65,8 \mathrm{~s}$.

$$
\begin{gathered}
I_{S P}=\frac{I_{T}}{m g}=\frac{98,7 \mathrm{Ns}}{0,153 \mathrm{~kg} \times 9,81 \mathrm{~m} / \mathrm{s}^{2}} \\
=65,8 \mathrm{~s}
\end{gathered}
$$

Finalmente, la Tabla 3 resume la caracterización y clasificación del motor cohete basada en los resultados arrojados por la prueba estática de combustión. Como se pudo corroborar, el desempeño del motor cohete concuerda con las especificaciones de diseño conceptual previamente establecidas (motor cohete clase $\mathrm{G}$ ).

Tabla 3. Caracterización y clasificación del motor cohete

\begin{tabular}{|l|c|}
\hline \multicolumn{1}{|c|}{ Característica } & Resultado \\
\hline Masa de grano propelente & $0,153 \mathrm{~kg}$ \\
\hline Empuje máximo $\left(\mathrm{F}_{\max }\right)$ & $227 \mathrm{~N}$ \\
\hline Impulso total $\left(\mathrm{I}_{\mathrm{T}}\right)$ & $98,7 \mathrm{~N}-\mathrm{s}$ \\
\hline Tiempo de propulsión $(t)$ & $1,3 \mathrm{~s}$ \\
\hline Empuje promedio $\left(\mathrm{F}_{\mathrm{avg}}\right)$ & $75,9 \mathrm{~N}$ \\
\hline Impulso especifico $\left(\mathrm{I}_{\mathrm{SP}}\right)$ & $65,8 \mathrm{~s}$ \\
\hline Clasificación del motor & Clase $\mathrm{G}$ \\
\hline
\end{tabular}

Fuente: elaboración propia.

Estos resultados son considerados de suma importancia para lograr la trasferencia tecnológica de este tipo de motores cohete y contribuir al desarrollo del campo de la cohetería experimental en Colombia, cuyas bases más importantes a nivel mundial se han logrado establecer a partir del diseño, construcción y lanzamiento de cohetes propulsados por combustibles sólidos.

\section{Conclusiones}

El desarrollo de esta investigación demostró la posibilidad de diseñar un motor cohete propulsado por un combustible sólido tipo Candy $\mathrm{KNSU}\left(65 \% \mathrm{KNO}_{3}-\right.$ $35 \%$ Sucrosa), siguiendo la metodología SRM, para su implementación en el lanzamiento de cohetes experimentales.

La validación del diseño del motor cohete, a través de una prueba real de combustión, permitirá escalar estos resultados en el desarrollo de nuevas versiones con mayores capacidades de propulsión.

Estos resultados impulsan el desarrollo de toda una línea de investigación enfocada en la optimización del desempeño de motores cohetes de combustibles sólidos, que a su vez fomente el desarrollo de misiones de cohetería experimental en Colombia.

Los resultados experimentales obtenidos por medio de la prueba estática de combustión permitieron concluir que el impulso total $\left(\mathrm{I}_{\mathrm{T}}\right)$ e impulso especifico $\left(\mathrm{I}_{\mathrm{S}}\right)$ del motor cohete fueron de 98,7 Ns y 65,8 s, respectivamente, con un valor de empuje promedio $\left(\mathrm{F}_{\mathrm{avg}}\right)$ de $75,9 \mathrm{~N}$ y un tiempo de propulsión de 1,3 s.

Los resultados experimentales permitieron clasificar el motor cohete diseñado como un motor clase $\mathrm{G}$, con un empuje máximo de $227 \mathrm{~N}$, destacando la obtención de este desempeño con la utilización de un combustible sólido tipo Candy KNSU de 0,153 kg de masa.

La metodología SRM (Solid Rocket Motor Design) resultó ser coherente con los resultados reales obtenidos mediante la prueba de combustión estática.

\section{Agradecimiento}

Los autores, miembros del Grupo de Investigación en Estudios Aeroespaciales (GIEA) de la Fuerza Aérea Colombiana, agradecen a la Sección de Investigación (SEINV) y al Grupo Académico (GRUAC) de la Escuela Militar de Aviación (EMAVI) por todo el apoyo recibido en el marco de esta investigación.

\section{Referencias}

[1] NASA, "Descripción general del programa Rocket de sondeo de la NASA: Oportunidades únicas para investigación científica enfocada, de bajo costo y rápida", NASA Rocking Science Rocking, 2019. [En línea]. Disponible en: https://rscience.gsfc.nasa.gov/srrov.html. 
[2] V. Marcado et al., "Progresos alcanzados en el proyecto universitario cohete sonda ULA", Universidad, Cienc. y Tecnol., vol. 13, no. 53, pp. 305-316, 2009.

[3] E. A. Acosta Vasquez, L. E. Marin, O. Jaimes, "Cohetes hidraulicos. Diseño, construccion y experimentacion, una experiencia integradora", Revista científica, vol. 13, no. 1, pp. 229-231, 2011, doi: $10.14483 / 23448350.966$

[4] M. Bandecchi, B. Melton, F. Ongaro, "Concurrent engineering applied to space mission assessment and design", Esa Bull. Sp. Agency, vol. 99, pp. 34-40, 1999.

[5] C. E. Álvarez Calderón, C. G. Corredor Gutiérrez, El espacio exterior: una oportunidad infinita para Colombia. Bogotá D.C.: Fuerza Aérea Colombiana, 2019.

[6] C. Seisdedos Corteguera, "Diseño y contrucción de un motor cohete hibrido", tesis de maestría, Universidad Carlos III de Madrid, 2013.

[7] J. Murcia Piñeros, S. Gómez Salcedo, "Diseño de los motores cohetes de propelente solido para el despegue asistido de un vehículo aéreo no tripulado (VANT)", Cienc. y Tecnol. Aeronáutica, vol. 17, pp. 29-39, 2012.

[8] D. Martinez Pino, "Diseño y optimización de un cohete de sondeo", trabajo fin de grado, Universidad de Sevilla, 2014.

[9] G. P. Suton, O. Biblarz, Rocket Propulsion Elements, Eighth Edi. New Jersey: John Wiley \& Sons, INC., 2010.

[10] L. Lacruz, M. Parco, R. Santos, C. Torres, J. Ferreira, P. Benítez-Díaz, "Análisis experimental de las oscilaciones de presión interna en un motor de combustible sólido para cohete sonda", Rev. Cienc. $e$ Ing., vol. 37, no. 2, pp. 81-88, 2016.

[11] C. Cubides, "Propelentes solidos: Una experimentacion para el futuro", Rev. Cienc. y Tecnol. del Ejercito, vol. 6, no. 12, pp. 11-19, 2015.

[12] R. Nakka, "Richard Nakka's Experimental Rocketry Web Site", 1997. [En línea]. Disponible en: http://www.nakka-rocketry.net/.
[13] R. A. Robayo-Salazar, "Cohetes tipo sonda con fines científicos, tecnológicos y militares: una oportunidad para su desarrollo en la EMAVI-FAC", en Ciencia, Gestión e Ingeniería en el Desarrollo Aeroespacial, Cali, Colombia, 2019, pp. 49-72.

[14] M. Correal, D. Castaño, O. Rincón, H. Aguillón, "Diseño y construcción de un cohete amateur tipo G", trabajo de grado, Universidad de San Buenaventura, 2009.

[15] E. A. Perez Reina, N. Bermeo Perez, D. A. Fuquen, "Diseño y construccion de un motor cohete que produzca 800 newton de empuje", trabajo de grado, Universidad de San Buenaventura, 2010.

[16] F. A. Riveros Enciso, L. A. Rodriguez Herrera, "Diseño y construcción de un cohete aficionado controlado mediante el accionamiento de una tobera de empuje vectorial", trabajo de grado, Universidad Militar de Nueva Granada, 2010.

[17] J. A. Aponte Rodriguez, D. Amaya Hurtado, A. Rubiano Fonseca, V. Prada Jimenez, "Modelado, diseño y construccion de un sistema activo de control de estabilidad de bajo costo para cohetes experimentales aficionado", Cienc. e Ing. Neogranadina, vol. 20, no. 1, pp. 77-96, 2010.

[18] J. A. Urrego Peña, F. A. Rojas Mora, "Misiones de coheteria experimental con propelente solido: Mision Seneca, Cohete AINKAA 1", Cienc. $e$ Ing. Neogranadina, vol. 20, no. 2, pp. 53-64, 2010.

[19] J. O. Murcia Piñeros, H. D. Ceron Muñoz, S. A. Gomez Salcedo, S. N. Pachon Laiton, "Diseño conceptual, preliminar y análisis de la trayectoria de vuelo de un cohete sonda de propelente solido para carga util de 2 kg", en Cuarto Congreso Internacional en Ciencia y Tecnología Aeroespacial (CICTA 2012), no. Octubre, pp. 1-11.

[20] O. D. Salazar Ceballos, M. A. Solorzano Gomez, "Diseño conceptual, preliminar y detallado del cohete sonda recuperable 'Aristarco I' propulsado con propelente solido", trabajo de grado, Fundacion Universitaria Los Libertadores, 2016. 
[21] N. Alvarez Rojas, J. Huérfano, O. Ojeda, "Diseño e implementación de misión para el lanzamiento de un cohete para tres kilometros de altura", en VIII Congreso Argentino de Tecnología Espacial, 2015, pp. 1-10.

[22] F. A. Galindo Herrera, J. O. Calderon Rojas, "Diseño estructural y aerodinámico de un cohete sonda para ser usado en la adquisición de variables atmosféricas", en XII Congreso Iberoamericano de Ingeniería Mecánica (CIBIM XII), 2015, pp. 1-9.

[23] D. Valdivieso Merino, M. A. Rojas Nova, A. F. Correa Rivera, "Diseño y validacion de los calculos de un motor cohete R-Candy E65", Research Report, Universidad Industrial de Santander, 2017.

[24] DPN Colombia, Política de desarrollo espacial: condiciones habilitantes para el impulso de la competitividad nacional. Bogotá, D.C. Colombia, Documento CONPES 3983, 2020.

[25] R. Nakka, "Solid propellant rocket motor desing and testing", trabajo de grado, University of Manitoba, 1984. 